\title{
Improved Aerodynamic Analysis for Hybrid Wing Body Conceptual Design Optimization
}

\author{
Frank H. Gern ${ }^{1}$ \\ NASA Langley Research Center, Hampton, VA 23681
}

\begin{abstract}
This paper provides an overview of ongoing efforts to develop, evaluate, and validate different tools for improved aerodynamic modeling and systems analysis of Hybrid Wing Body (HWB) aircraft configurations. Results are being presented for the evaluation of different aerodynamic tools including panel methods, enhanced panel methods with viscous drag prediction, and computational fluid dynamics. Emphasis is placed on proper prediction of aerodynamic loads for structural sizing as well as viscous drag prediction to develop drag polars for HWB conceptual design optimization. Data from transonic wind tunnel tests at the Arnold Engineering Development Center's 16-Foot Transonic Tunnel was used as a reference data set in order to evaluate the accuracy of the aerodynamic tools. Triangularized surface data and Vehicle Sketch Pad (VSP) models of an X-48B 2\% scale wind tunnel model were used to generate input and model files for the different analysis tools. In support of ongoing HWB scaling studies within the NASA Environmentally Responsible Aviation (ERA) program, an improved finite element based structural analysis and weight estimation tool for HWB center bodies is currently under development. Aerodynamic results from these analyses are used to provide additional aerodynamic validation data.
\end{abstract}

\section{Nomenclature}

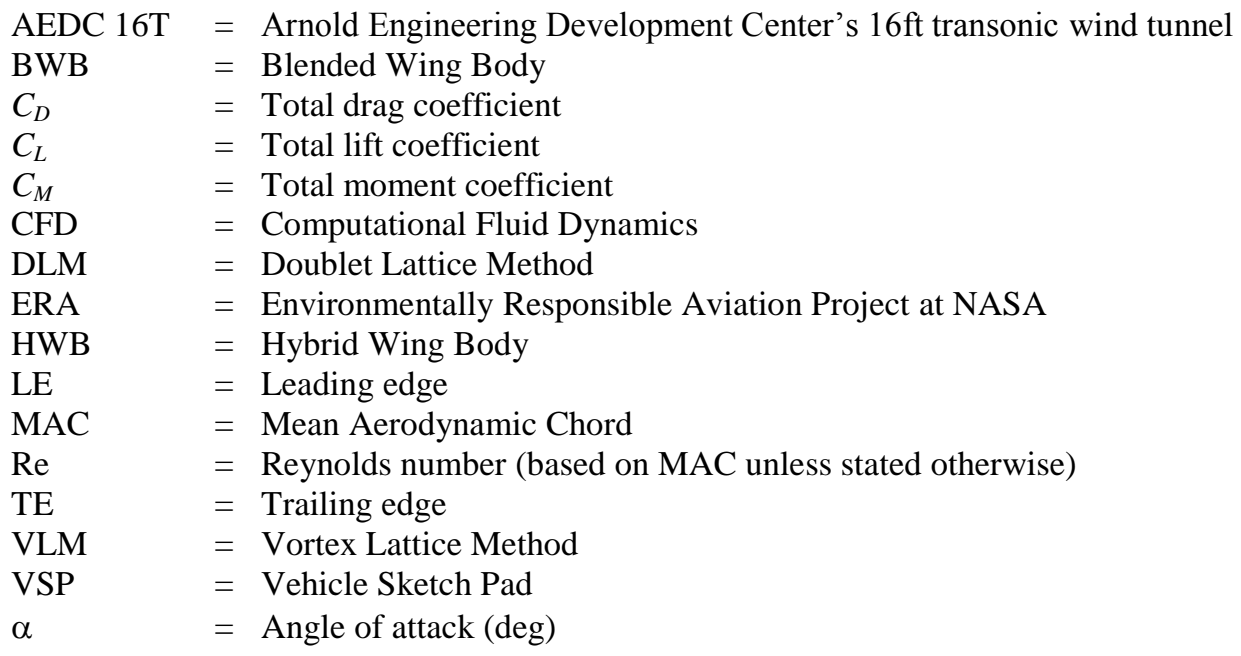

\section{Introduction}

ЧYBRID wing body (HWB) or Blended Wing Body (BWB) aircraft designs have been considered promising alternatives to conventional tube and wing aircraft designs due to their large potential fuel savings and increased aerodynamic efficiency. ${ }^{1}$ Fuel burn reductions of 25\% and higher have been published for some ultra-high capacity configurations carrying up to 800 passengers. Most notable is the work of Liebeck and his co-workers at

\footnotetext{
${ }^{1}$ Senior Aerospace Engineer, Aeronautics Systems Analysis Branch.
} 
the Boeing Company. Their 450 passenger BWB-4501L design recently resulted in the $8.5 \%$ scale $\mathrm{X}-48 \mathrm{~B}$ flight demonstrator (Fig. 1). ${ }^{2}$

A significant difficulty in dealing with HWB design optimization has always been the lack of a data base of known "flying" designs which may serve as calibration and validation points for optimization programs like FLOPS, especially when compared to the vast number of available tube and wing aircraft data. When transitioning from the conceptual to the preliminary design phase, the aircraft designer needs to be sure that the design chosen for further optimization is actually a viable design, and as a result of the lack of validation cases, the development

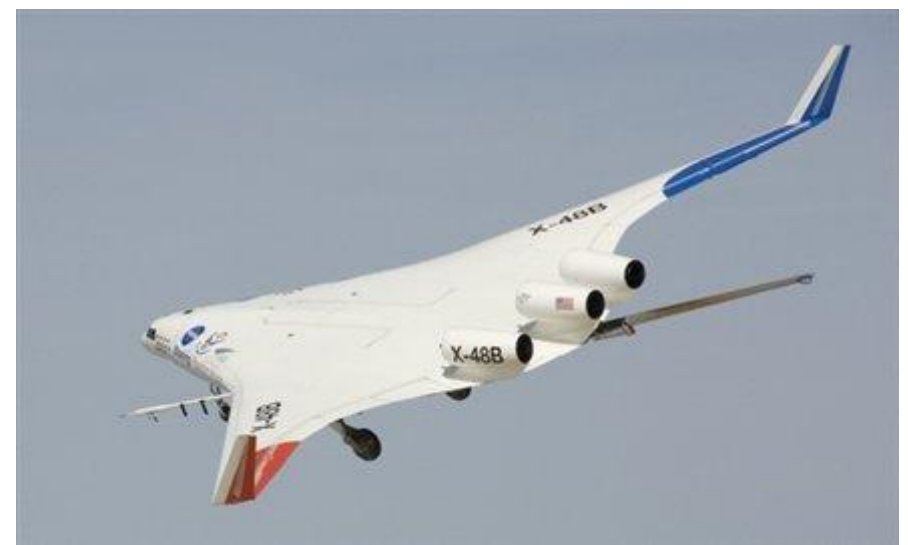

Figure 1: Boeing's X-48B Blended Wing Body flight demonstrator (Boeing picture). of improved fidelity analysis tools becomes imperative already for the conceptual design loop.

Therefore, in order to validate the projected fuel burn and noise reduction potential of HWB designs for NASA's Environmentally Responsible Aviation Program (ERA), significant efforts have been put forward to develop advanced structural and aerodynamic analysis tools for HWB conceptual design optimization. Recently developed tools include finite element (FEM) based structural analyses to provide enhanced capabilities for HWB center body structural sizing and weight estimation. Aerodynamic methods improvement has been geared towards increased fidelity in-the-loop methods like enhanced panel codes and computational fluid dynamics (CFD).

While the use of CFD methods to explore large design spaces is still limited by available computation power and reasonable turn-around times, several promising alternatives like enhanced panel methods with viscous drag prediction capabilities have become candidate tools for HWB conceptual design optimization. This paper describes recent efforts at NASA Langley's Aeronautics Systems Analysis Branch towards the evaluation and validation of different aerodynamic tools ranging from panel codes to Euler and Navier-Stokes CFD methods. Significant lowspeed and transonic wind tunnel testing at the NASA Langley National Transonic Facility (NTF) and the Arnold Engineering Design Center 16ft Transonic Tunnel (AEDC-16T) preceded flight testing of the X-48B and resulted in a wealth of available aerodynamic test data. Validation data for the present study is based on a June 2007 transonic test in the AEDC-16T on a 2\% scale model of Boeing's BWB-450-1L design.

\section{Aerodynamic Modeling and Analysis}

For aerodynamic modeling and validation, wind tunnel model data of the Boeing X-48B 2\% scale model was used to generate geometry and input files for the different aerodynamic tools. Several different models were generated to export the X-48B geometry into required formats for the various analysis packages (Table 1). The greatest challenge was that each analysis tool requires different sets of input parameters, with different input file formats.

Table 1: Summary of investigated aerodynamics codes.

\begin{tabular}{lllc}
\hline \hline Code name & Code type & Remarks & Reference \\
\hline Vorlax & Panel code, VLM & Includes thick leading edge correction & 3 \\
Vorview & Panel code, VLM & Graphical front end for Vorlax & 4 \\
MSES & 2-D Euler & Stripwise airfoil drag prediction & 5 \\
CPPAero & Enhanced panel code, VLM & Includes stripwise shock and viscous drag & 6 \\
Nastran & Panel code, DLM & Included in structural model for loads & 7 \\
Cart3D & CFD, 3-D Euler & Used as scaling reference for full scale configuration & 8 \\
Fun3D & CFD, 3-D Navier-Stokes & Used as scaling reference for full scale configuration & 9 \\
\hline \hline
\end{tabular}




\section{A. Model Development}

As a first step, triangularized surface data of the X-48B wind tunnel model was imported into Matlab to generate suitable input files for the different analysis tools. Model data provided by the NASA Langley Configuration Aerodynamics Branch is a Tecplot data file of the $2 \%$ scale wind tunnel model of the Boeing BWB-450-1L with a wingspan of $4.80 \mathrm{ft}$ and a centerline chord length of $3.13 \mathrm{ft}$. The model data is given by a set of unstructured surface grid data points from triangular panels (Fig. 2). ${ }^{10}$

A Matlab routine was developed to generate hermite files for Vorview. To provide a cleaner surface for initial code validation of the vortex lattice panel

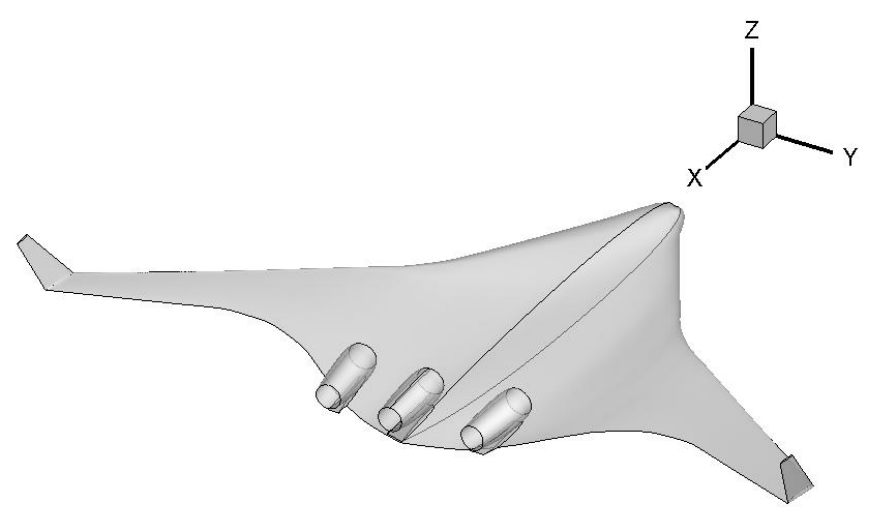

Figure 2: Tecplot model of the $\mathrm{X}-48 \mathrm{~B} 2 \%$ scale rotary wind tunnel model. methods, engine data was removed from the slices. The slice data was written to a text data file in the Vorview hermite format. To enhance portability of the model data to other codes available in the NASA Langley Aeronautics Systems Analysis Branch and to generate geometry files for the CFD codes, a VSP ${ }^{11}$ model was developed from the extracted airfoil stack data. An overall modeling summary is shown in Fig. 3.

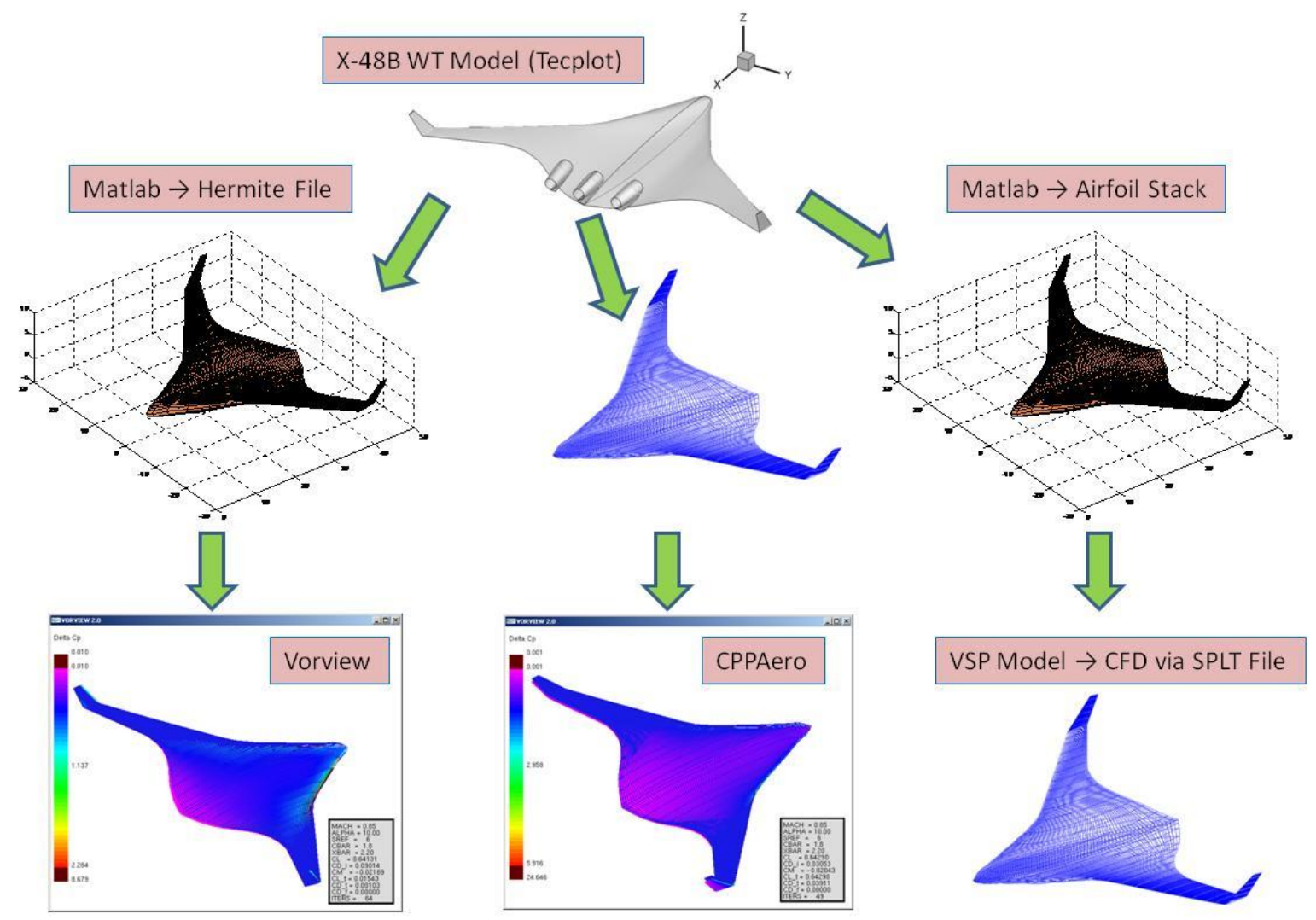

Figure 3: Aerodynamic codes validation modeling overview. 


\section{B. HWB Nastran Model}

The HWB Nastran model has been developed in support of the HWB scaling study presented by Nickol ${ }^{12}$ to investigate the viability of a hybrid wing body airplane for smaller commercial transport aircraft down to regional jet size (about 100 passengers). In an effort to improve previous FEM and FLOPS based analysis methods for weight estimation of the HWB center body, a full aeroelastic finite element model of an HWB primary structure has been implemented. To maintain the capability to interface with FLOPS, most of the structural parameters for finite element modeling of the HWB structure are based on the center body home plate analogy introduced by Nickol and McCullers. ${ }^{13}$

As part of the aeroelastic validation of the structural model, Nastran aerodynamic loads have been compared to Vorlax and CPPAero results. The Nastran model is generated by exporting the VSP surface model and extracting a parametric representation of the HWB primary structure from the exported data, which is then used to build a Nastran bulk data file (Figs. 4 and 5). More details regarding the structural HWB finite element modeling in Nastran can be found in Ref. 14.
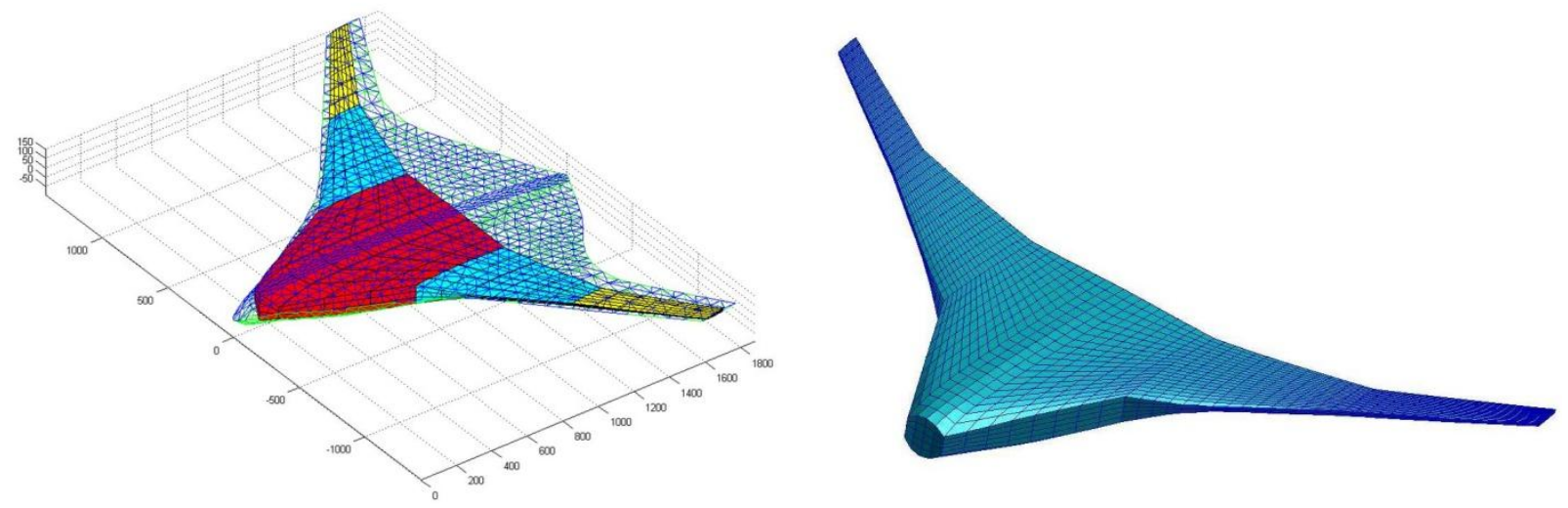

Figure 4: Left: Exported VSP surface data with overlay of HWB primary structure. Right: Patran rendering of the generated Nastran bulk data file.

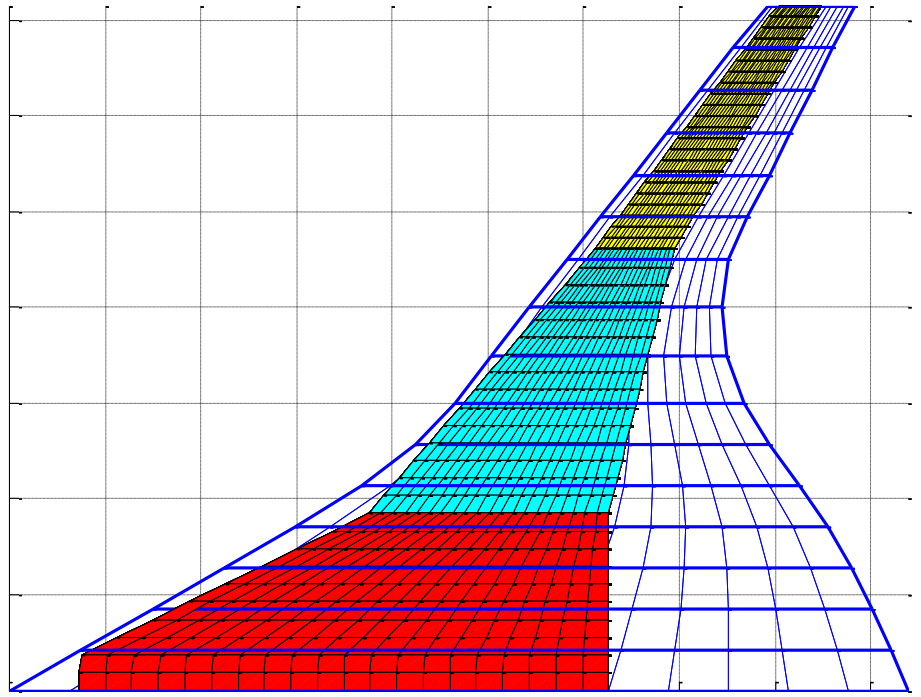

Figure 5: HWB Primary structure with overlay of Nastran doublet lattice aerodynamic paneling. 


\section{Reference Wind Tunnel Data Set}

In June 2007, the X-48B 2\% scale model of the BWB-450-1L was tested at the Arnold Engineering Development Center (AEDC) 16T wind tunnel. The test was called TF1038 under the AEDC designation system. As a result of this test, sufficient data was generated to develop a transonic aerodynamic database for vehicle lift, drag, and moment coefficients vs. angle of attack and Mach number. ${ }^{15}$ The data set used for this analysis was collected at Mach numbers ranging from 0.5 to 0.85 and MAC based Reynolds numbers of 10 million. Complete details of the AEDC T1038 test are given in Ref. 15. Results for vehicle $C_{L}, C_{M}, C_{D}$, and drag polars vs. Mach number are shown in Fig. 6.

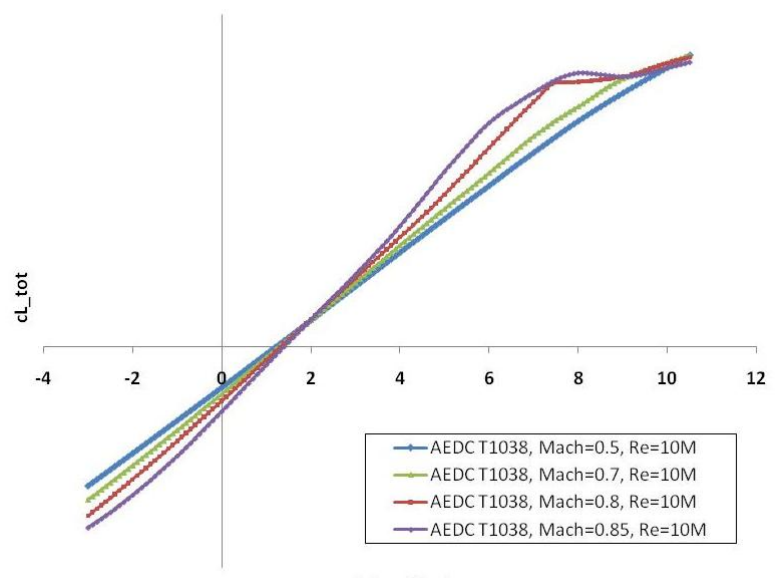

alpha (deg)

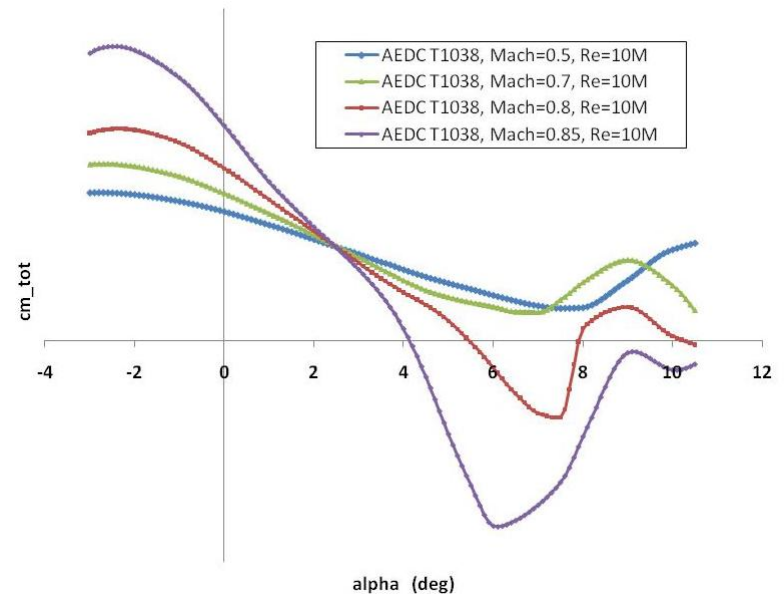

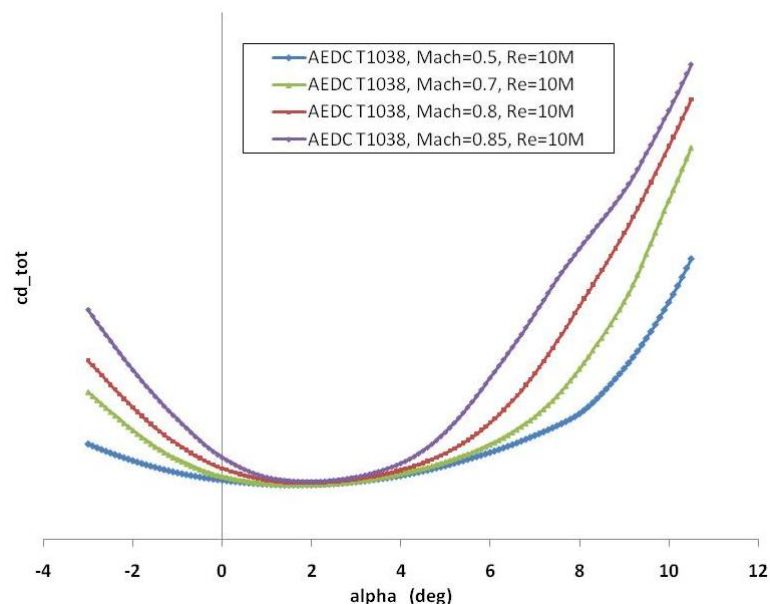

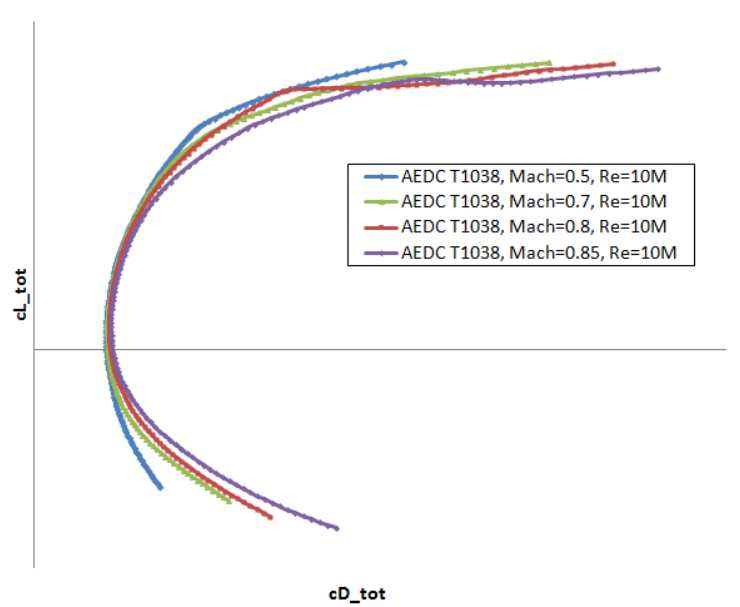

Figure 6: Vehicle baseline aerodynamic coefficients from AEDC 16T transonic wind tunnel test.

\section{Aerodynamic Validation Results: Panel Codes}

Vorlax is a generic vortex lattice panel code for subsonic and supersonic flow applications. To improve its user friendliness, the graphic front end Vorview has been wrapped around the original Vorlax solver. MSES was used for viscous drag predictions within Vorview. CPPAero is a VLM based aerodynamics analysis tool developed by Avid LLC in Yorktown, VA specifically for BWB configurations. The program uses Vorlax as a general VLM solver but has several additional, partly BWB specific features to account for compressibility and transonic effects, thickness effects, viscous drag, and BWB specific S\&C issues. Through a built-in XML loader, an XML file can be imported into CPPAero in order to generate the input and geometry files required to run a CPPAero analysis. 


\section{A. Lift Coefficients}

Figure 7 shows lift coefficient comparisons between wind tunnel data, Vorlax, and CPPAero runs for the X-48B $2 \%$ scale rotary wind tunnel model. Both low speed and transonic data sets agree exceptionally well with wind tunnel data in the linear region of the lift gradient. As a linear method, Vorlax does not predict the transonic lift rise nor flow separation at higher angles of attack. CPPAero's built-in corrections capture but overpredict the transonic lift rise up to pitch angles of stall onset.

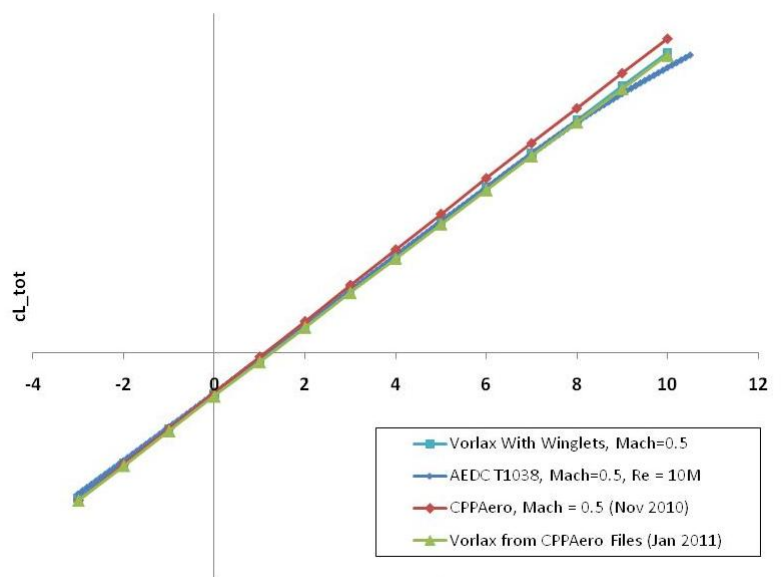

alpha (deg)

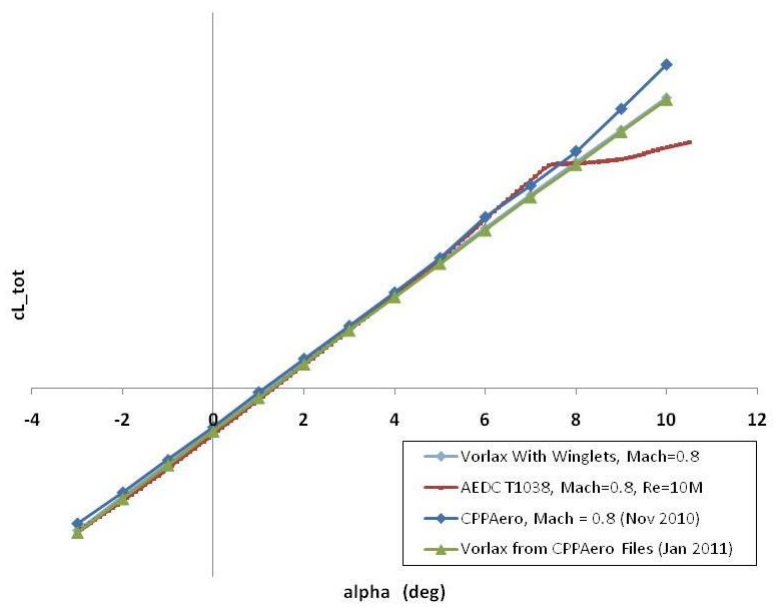

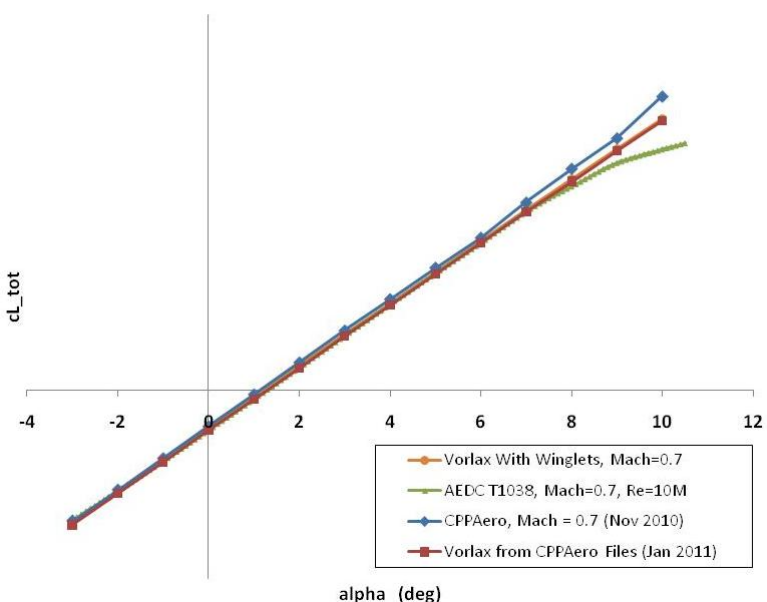

alpha (deg)

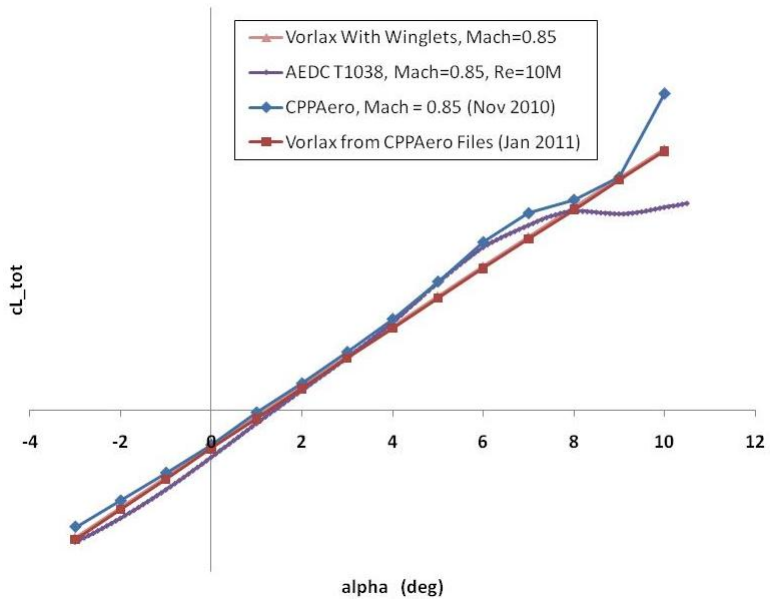

alpha (deg)

Figure 7: Lift coefficients vs. pitch angle and Mach number for the investigated panel codes.

\section{B. Drag Polars}

Drag polars from all panel codes were compared to experimental data for the same Mach number range (Fig. 8). In general, panel codes only predict induced drag and portions of pressure drag related to airfoil camber. Therefore, different strategies have been used to improve drag predictions by including viscous drag calculations. The general approach for this process usually solves the linear VLM problem and then uses the VLM based pressure distribution to compute viscous drag contributions.

CPPAero uses a zonal approach where the induced drag at a given subsonic/transonic condition is calculated using the incompressible drag polar shape as its basis. The wing Oswald efficiency factor is found from the incompressible Vorlax results. This is modified as necessary for the flow zones on each wing section (see Ref. 6).

MSES uses 2D airfoil data to generate sectional viscous drag predictions which are summed up in the spanwise direction to provide the total viscous drag. Depending on the airfoil geometry and Mach/Reynolds number conditions, MSES has shown to have varying convergence characteristics. At lower Mach numbers, Vorview results with MSES viscous drag show excellent agreement with test data. For transonic Mach numbers, MSES convergence was sketchy, no reasonable result could be obtained for the Mach 0.85 condition. 

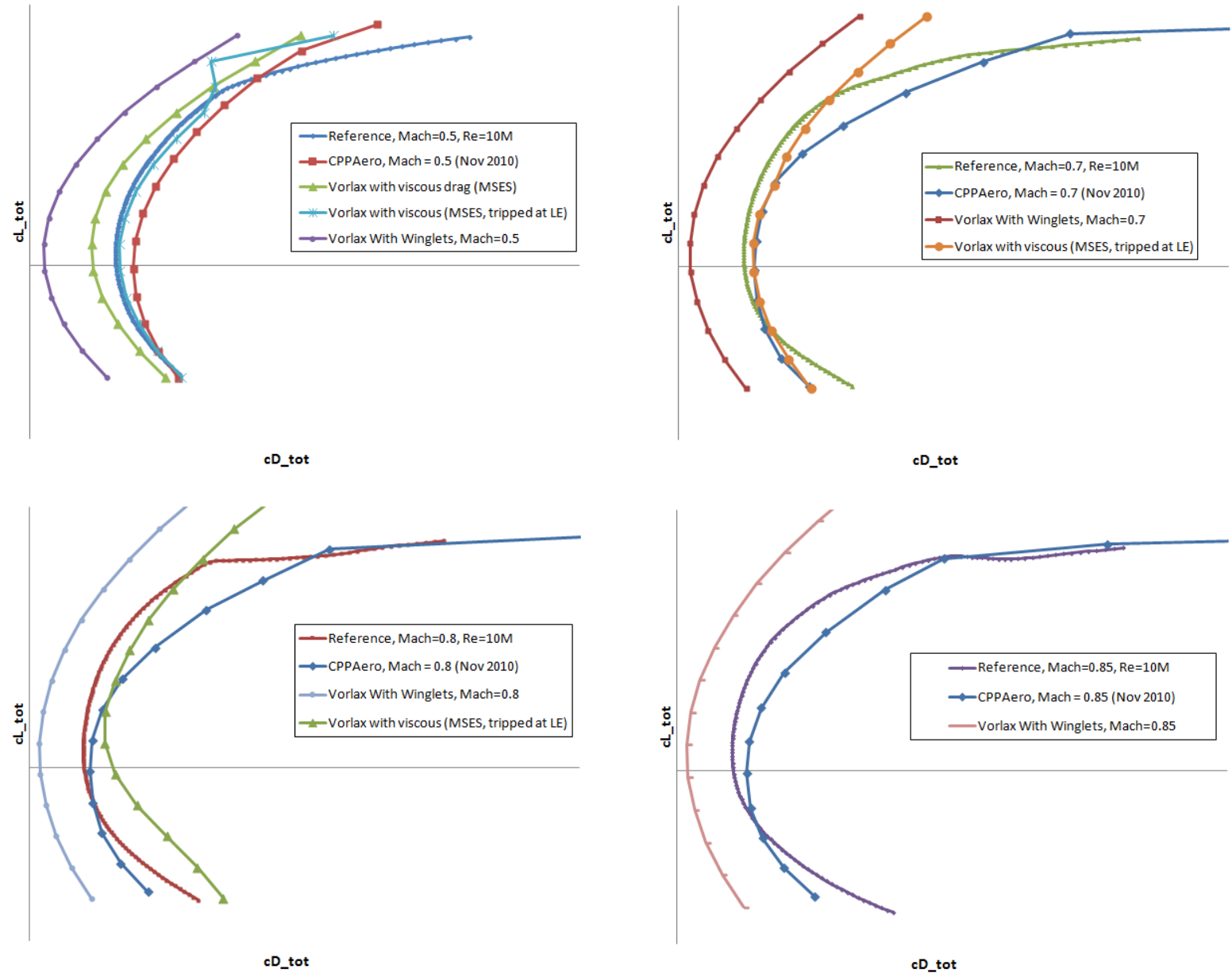

Figure 8: Drag polars vs. Mach number for the investigated panel codes.

\section{Spanload Validation Using Nastran}

From the wind tunnel test data, only total lift, moment and drag coefficients were available. Spanload distributions for aerodynamics loads predictions were therefore validated by comparing vortex lattice Vorview results with spanwise lift coefficients obtained from Nastran doublet lattice aerodynamics using the HWB structural model described in section II.B and Ref. 14.

Figure 9 shows excellent agreement between both methods for several pitch angles ranging from 1 to 4 degrees. The Vorview spanload data is based on rigid lifting surfaces since Vorview does not employ an underlying structural model. Therefore, only rigid sectional lift coefficients from the Nastran solution are shown for the comparison.

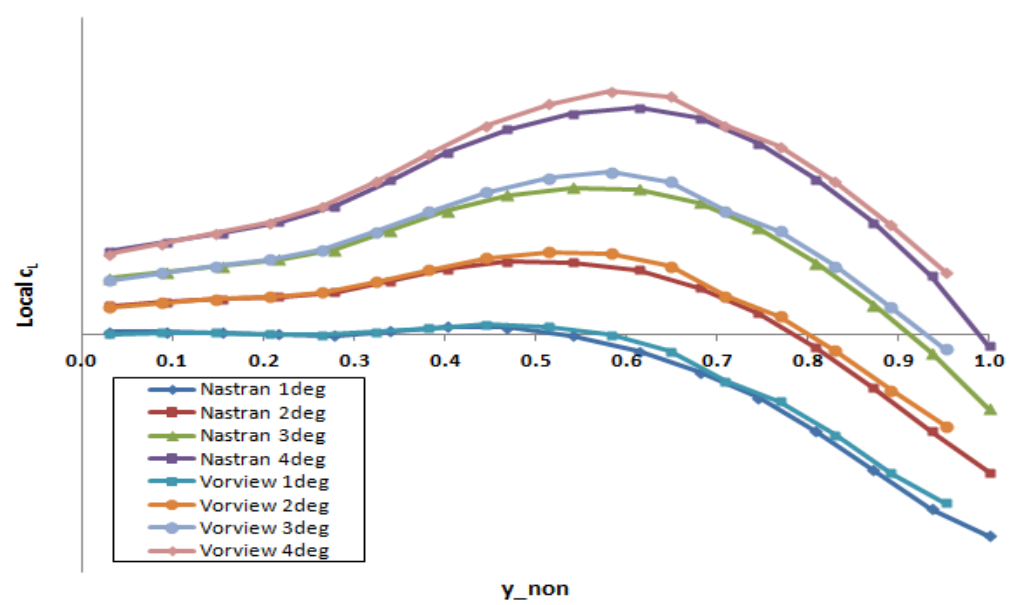

Figure 9: Vorlax vs. Nastran spanload comparison. 


\section{Aerodynamic Validation Results: CFD Codes}

Although not considered for direct use in a conceptual design and optimization loop due to computation power and turn-around time constraints, two CFD codes have been assessed in an effort to generate data for Reynolds number scaling from wind tunnel model dimensions to full scale HWB designs. Cart3D is a high-fidelity inviscid analysis package for conceptual and preliminary aerodynamic design. It allows users to perform automated CFD analysis on complex geometries. The package includes utilities for geometry import, surface modeling and intersection, mesh generation, flow simulation and post-processing of results. ${ }^{8}$

Fun3D is an unstructured viscous CFD code solving the three-dimensional Navier-Stokes equations. The solver has been applied to a number of complex large-scale problems by groups at NASA, industry, and academia. Internally, the software has been used to study airframe noise, space transportation vehicles, flow control devices using synthetic jets, the design of wind tunnel and flight experiments, and so forth. Boeing, Lockheed, Cessna, New Piper, and others have used the tools for applications such as high-lift, cruise performance, and studies of revolutionary concepts. ${ }^{9}$

\section{A. Lift Coefficients}

As expected, results from both CFD codes show good agreement with tunnel data for the lift coefficients. However, it was noted that Cart3D significantly overpredicts the transonic lift increase at higher pitch angles. As a reference for the investigated panel codes lift coefficients from Vorlax are also included in the plots in Fig. 10.
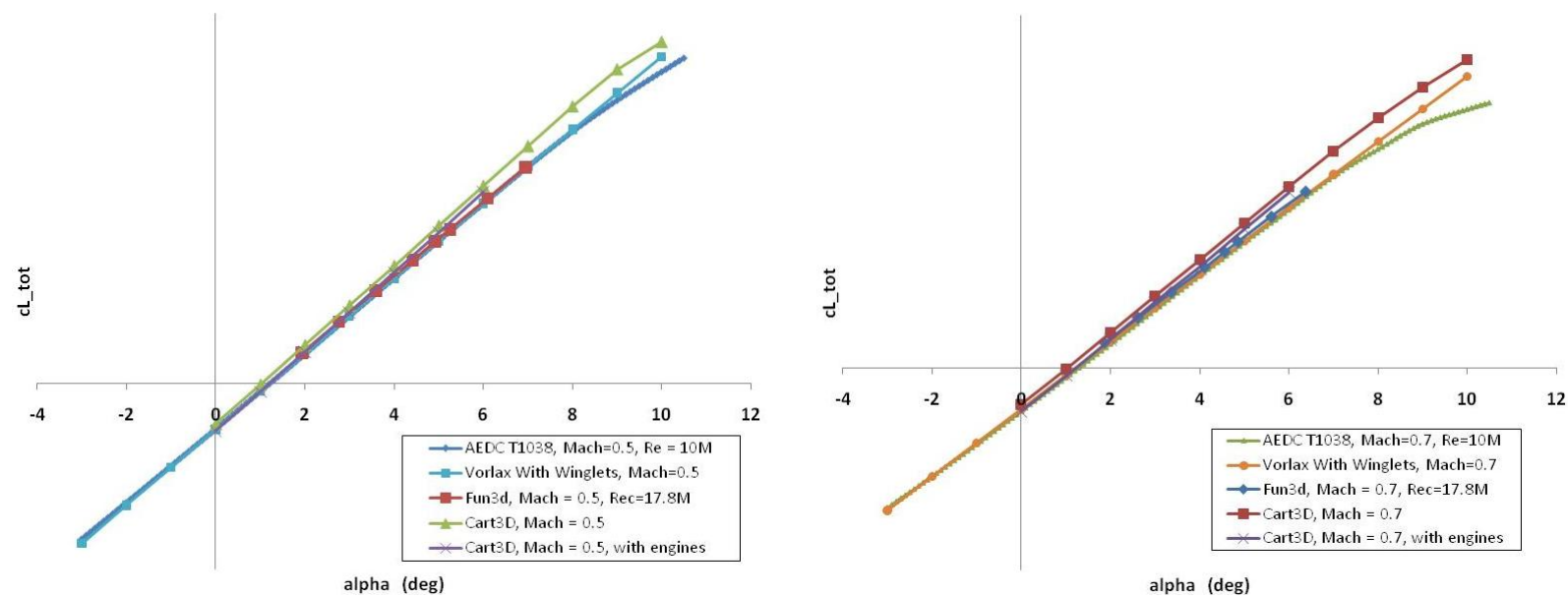

alpha (deg)
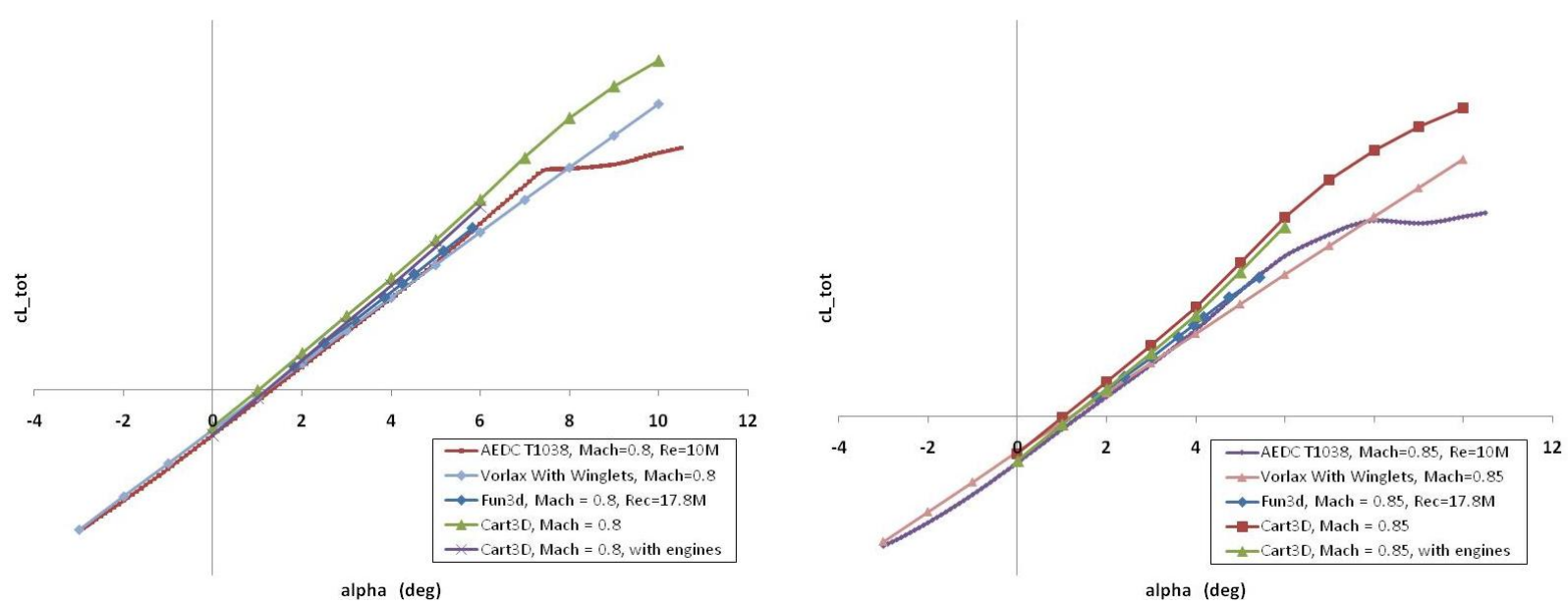

Figure 10: Lift coefficients vs. pitch angle and Mach number for the investigated CFD codes. 


\section{B. Drag Polars}

With Fun3D being the only viscous code in this assessment, the drag polar predicted by Fun3D shows the best agreement with test data. Pressure drag from Fun3D agrees well with Cart3D and the panel codes for all Mach numbers (Fig. 11).
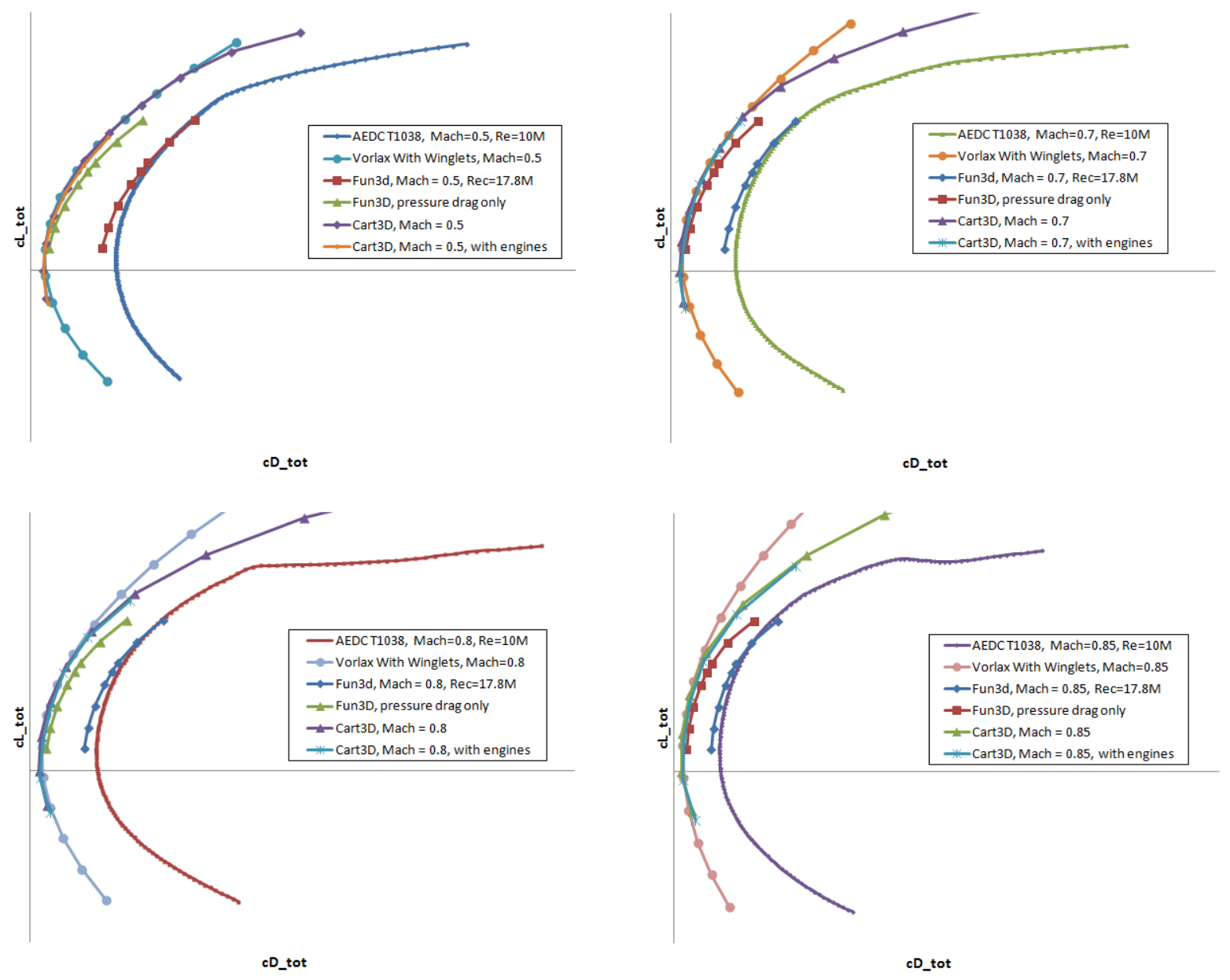

Figure 11: Drag polars vs. Mach number for the investigated CFD codes.

\section{Drag Polar Discussion - CFD vs. Enhanced Panel Methods}

Subsonic drag predictions of both CFD and panel codes agree well with tunnel data. At transonic Mach numbers, the enhanced panel methods fail to predict viscous drag contributions accurately enough for aircraft performance optimization. Since both enhanced panel methods are based on stripwise viscous drag calculations, discrepancies in viscous drag appear to be mainly due to the difficulty in accounting for three-dimensional relief effects which become prominent at transonic Mach numbers. CPPAero accounts for 3-D relief based on the analytical 3-D relief relations introduced by Hoerner ${ }^{16}$ and predicts total transonic drag trends more reasonably. As currently implemented, MSES viscous drag predictions are based on 2-D airfoil sectional drag with no 3-D relief. As a result, viscous drag is overpredicted at transonic Mach numbers.

\section{Viscous Drag Scaling}

As shown in the previous sections, lift and moment coefficients from Vorlax agree very well with wind tunnel data and Vorlax spanload distributions have been verified by Nastran doublet lattice aerodynamics. In order to use Vorlax results for aerodynamic performance optimization, a more accurate method of viscous drag prediction was 
derived with the goal of using induced drag numbers from Vorlax and adding viscous drag separately as a contribution to $c_{d 0}$. This way, aerodynamic lift variations due to planform changes can be easily captured through Vorview's geometry flexibility, while at the same time maintaining accurate viscous drag numbers without the necessity of excessive amounts of CFD runs.

In general, viscous drag $c_{d v}$ can be derived from skin friction drag $c_{d f}$ by the well-known relationship

$$
c_{d v}=c_{f} \frac{S_{w e t}}{S_{r e f}} f f_{H W B}
$$

In Eq. (1), $f f_{H W B}$ is a generic HWB form factor derived from CFD or wind tunnel data, while $S_{w e t}$ and $S_{\text {ref }}$ are the wetted and reference areas of the HWB, respectively.

A fundamental relationship for the skin friction $\operatorname{drag} c_{f}$ vs. Reynolds number has been presented by Hoerner as ${ }^{16}$

$$
c_{f}=K / \operatorname{Re}_{l}^{1 / m}
$$

Ref. 16 indicates that for a Reynolds number range between $10^{7}$ and $10^{9}$, the values $m=7$ and $K=0.030$ may be used. The resulting relationship between Reynolds number and skin friction coefficient $c_{f}$ is shown in Fig. 12 as a blue line. To account for compressibility effects, additional corrections to Hoerner's skin friction drag relations have been applied based on free stream Mach number ${ }^{17-19}$, where

$$
c_{f c o m}=\frac{1}{T} c_{f}
$$

with

$$
T=1+0.155 M^{2}
$$

To check the scalability of the presented approach for HWB viscous drag predictions, additional CFD runs were performed at different Reynolds numbers from subscale Reynolds numbers to full scale cruise flight conditions for the Boeing BWB450. CFD results match the Hoerner equation extremely well across the entire Reynolds number range from $5 \mathrm{M}$ to $200 \mathrm{M}$, i.e. covering both the $\mathrm{X}-48 \mathrm{~B} 2 \%$ scale wind tunnel model and BWB450 full scale cruise conditions (see dashed lines in Fig.12). Additional available CFD based viscous drag data for the BWB710 follows the same trend and is also labeled in Fig. 12.
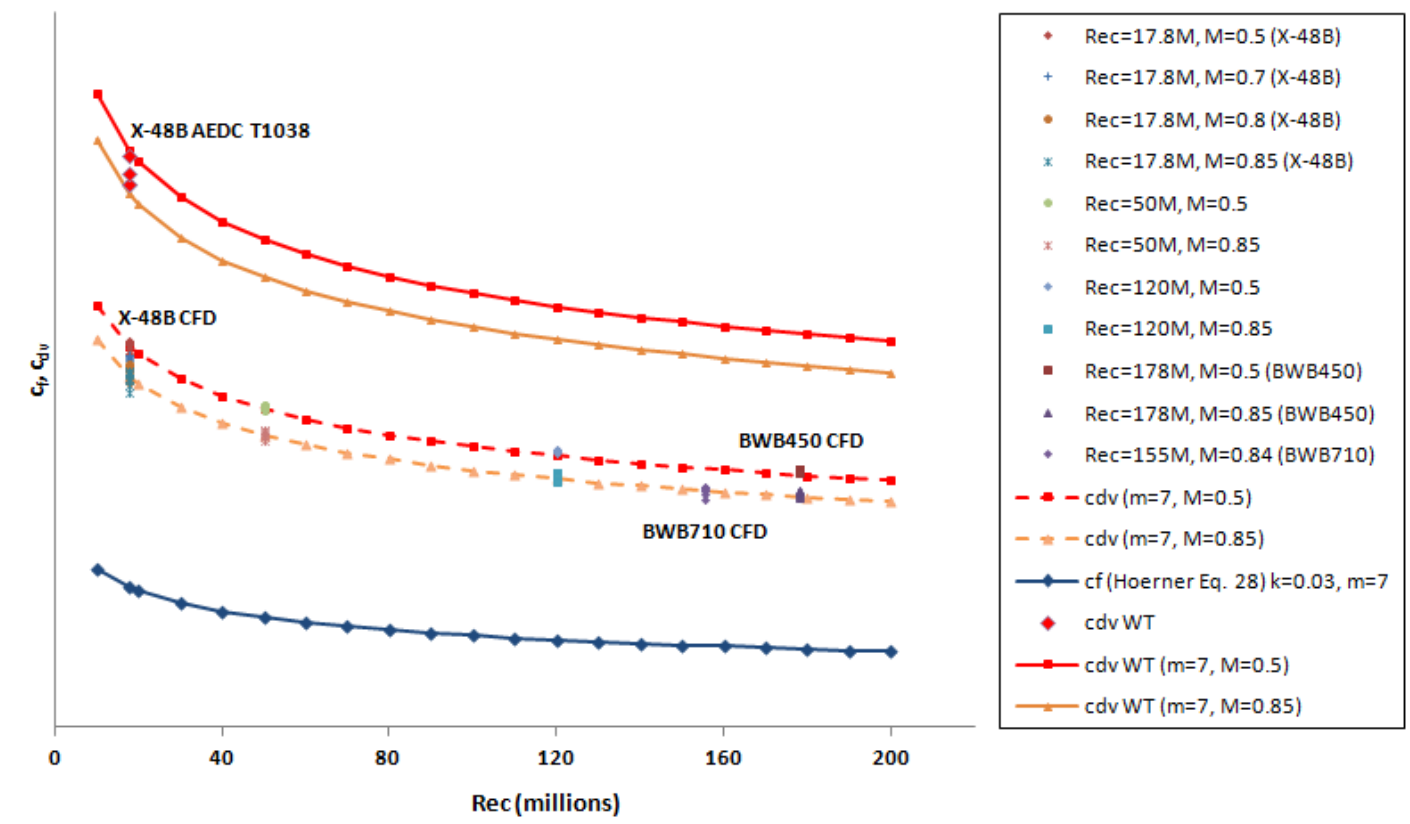

Figure 12: Skin friction drag and viscous drag coefficients vs. Reynolds number from CFD data and wind tunnel testing. 
Drag predictions from the $2 \%$ scale AEDC T1038 wind tunnel test show that viscous drag is slightly underpredicted by Fun3d. This is a common issue in viscous (and therefore total) drag prediction and may be attributed to wind tunnel installation effects, CFD turbulence models, differences in wind tunnel model vs. CFD model configuration etc.

HWB form factors $f f_{H W B}$ for Eq. (1) have been derived from both CFD viscous drag results and wind tunnel test data. Total drag coefficients vs. pitch angle as well as drag polars for both form factors are shown in Figs. 13 and 14.
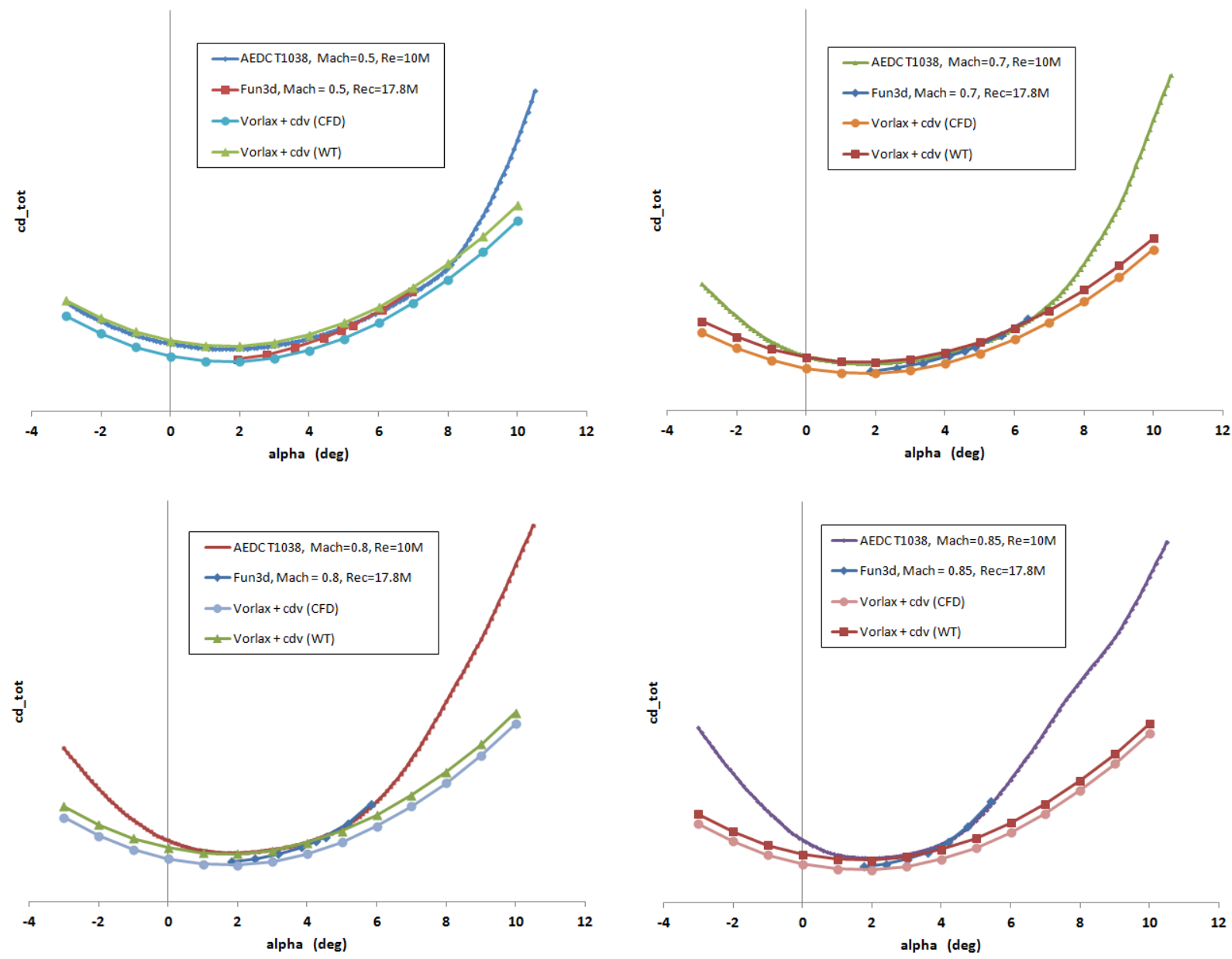

\section{Figure 13: Total drag coefficients vs. pitch angle for Vorlax with viscous drag (form factors derived from CFD and wind tunnel data).}

As expected, total drag predictions using CFD based form factors slightly underpredict wind tunnel drag measurements. Vorlax pressure drag with wind tunnel based viscous drag predictions shows excellent agreement with tunnel data even at transonic Mach numbers. While total drag predictions depart from tunnel data at higher pitch angles, predictions match tunnel data extremely well in the pitch angle and Mach number range relevant for aerodynamic performance optimization at cruise, as well as low speed take-off and landing conditions.

The Hoerner approach for predicting viscous drag for HWB configurations works well because the form factors derived from wind tunnel or CFD data are rather insensitive to planform geometry variations within this aircraft category. This method of calculating viscous and total HWB drag for aerodynamic performance optimization has been successfully applied by Nickol in his HWB scaling study. ${ }^{12}$ 

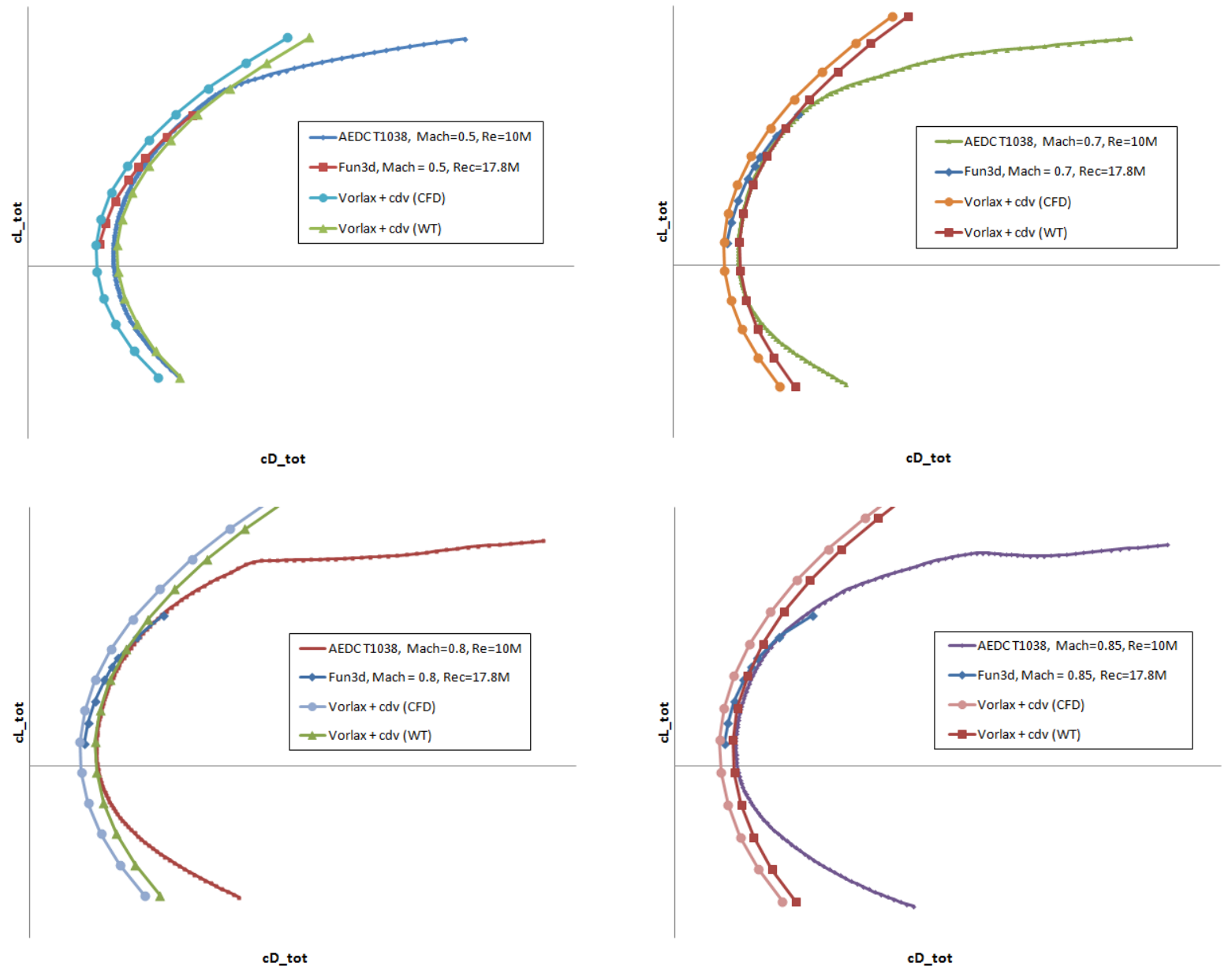

Figure 14: Drag polars for Vorlax with viscous drag (form factors derived from CFD and wind tunnel data).

\section{Conclusions}

Lift and moment predictions from both CFD and panel codes are suitable for loads calculations and HWB sizing. As expected, at transonic Mach numbers, fully viscous Navier-Stokes based CFD tools like Fun3D outperform enhanced panel methods and Euler codes in accurately predicting total drag polars for performance optimization, mainly due to the difficulty of accurately accounting for three-dimensional relief effects or viscous boundary layer effects. As of now, all of the investigated enhanced panel codes failed to provide accurate viscous drag predictions. Results obtained from CPPAero demonstrate that this problem may be overcome by implementing analytical 3-D relief procedures. However, while trends are predicted correctly, increased accuracy will still require tuning of the respective program parameters with wind tunnel or CFD data.

The use of a Reynolds number and Mach number based scaling scheme derived from the Hoerner relationships for predicting skin friction and viscous drag so far has shown the best results for viscous drag predictions apart from using fully viscous CFD analyses. The Hoerner approach works well for HWB configurations since form factors for viscous drag calculations are deemed to be rather insensitive to HWB planform geometry variations due to their overall aerodynamic simplicity and effectiveness. After all, for this very reason, HWB airplanes are being considered as alternatives to conventional tube and wing transport airplanes. ${ }^{1}$ 


\section{Acknowledgements}

The author would like to thank the following co-workers at NASA Langley for their contributions and valuable discussions: Craig L. Nickol, Andy Hahn, Doug Wells, Irian Ordaz, Beth Lee-Rausch, Robert McDonald, Dan Vicroy, and Melissa Carter.

The presented work was supported by Dr. Fay Collier under the NASA Environmentally Responsible Aviation (ERA) project.

\section{References}

1 Liebeck, R.H., "Design of the BWB Subsonic Transport", AIAA-2002-0002, 40 ${ }^{\text {th }}$ AIAA Aerospace Sciences Meeting and Exhibit, January 14-17, 2002, Reno, NV.

2 Risch, T., Cosentino, G., Regan, C.D., Kisska, M., and Princen, N., “X-48B Flight-Test Progress Overview,” AIAA-20090934, $47^{\text {th }}$ AIAA Aerospace Sciences Meeting and Exhibit, January 5-8, 2009, Orlando, Florida.

3 Miranda, L.R., Elliott, R.D., and Baker, W.M., A Generalized Vortex Lattice Method for Subsonic and Supersonic Flow Applications, NASA-CR-2865, Lockheed-California Company, Burbank, CA, December 1977.

4 Vorview Documentation, Vorview 1.7.4, 1999, contact: A. Hahn, Aeronautics Systems Analysis Branch, NASA Langley Research Center, Mail Stop 442, Hampton, VA 23681-0001.

5 Drela, M., "Newton Solution of Coupled Viscous/Inviscid Multielement Airfoil Flows," AIAA-1990-1470, 21 ${ }^{\text {st }}$ Fluid Dynamics, Plasma Dynamics and Lasers Conference, Seattle, WA, June 18-20, 1990.

6 CPPAero User's Guide, Avid LLC, Yorktown, VA, February, 2011.

7 Rodden, W.P. and Johnson, E.H. (Editors), MSC.Nastran Version 68, Aeroelastic Analysis User's Guide, MSC Software.

8 Cart3D Documentation, available online through NASA Ames Research Center, http://people.nas.nasa.gov/ aftosmis/cart3d/ 9 Fun3D Manual, available online at NASA Langley Research Center, http://fun3d.larc.nasa.gov/.

10 Vicroy, D.D., "Blended-Wing-Body Low-Speed Flight Dynamics: Summary of Ground Tests and Sample Results," AIAA2009-0933, 47 ${ }^{\text {th }}$ AIAA Aerospace Sciences Meeting and Exhibit, January 5-8, 2009, Orlando, Florida.

11 Hahn, A., "Vehicle Sketch Pad: A Parametric Geometry Modeler for Conceptual Aircraft Design," 48 ${ }^{\text {th }}$ AIAA Aerospace Sciences Meeting and Exhibit, January 4-7, 2010, Orlando, Florida.

12 Nickol, C.L., "Hybrid Wing Body Configuration Scaling Study," presented on the $50^{\text {th }}$ AIAA Aerospace Sciences Meeting, January 9-12, 2012, Nashville, Tennessee.

13 Nickol, C.L. and McCullers, L.A., "Hybrid Wing Body Configuration System Studies," AIAA-2009-0931, 47" AIAA Aerospace Sciences Meeting and Exhibit, January 5-8, 2009, Orlando, Florida.

14 Gern, F.H., "Improved Structural Analysis for Hybrid Wing Body Conceptual Design Optimization," accepted for 53rd AIAA/ASME/ASCE/AHS/ASC Structures, Structural Dynamics and Materials Conference, Honolulu, Hawaii, USA, 23-26 April 2012.

15 Carter, M.B., Vicroy, D.D., and Patel, D., "Blended-Wing-Body Transonic Aerodynamics: Summary of Ground Tests and Sample Results," AIAA-2009-0935, 47 ${ }^{\text {th }}$ AIAA Aerospace Sciences Meeting and Exhibit, January 5-8, 2009, Orlando, Florida.

16 Hoerner, S.F., Fluid-Dynamic Drag, Practical Information on Aerodynamic Drag and Hydrodynamic Resistance, Hoerner Fluid Dynamics, P.O. Box 21992, Bakersfield, CA, 1965.

17 Kulfan, B.M., “Assessment of CFD Predictions of Viscous Drag,” AIAA-2000-2391, Fluids 2000, June 19-22, 2000, Denver, Colorado.

18 Peterson, J.B., A Comparison of Experimental and Theoretical Results for the Compressible Turbulent-Boundary-Layer Skin Friction With Zero Pressure Gradient, NASA-TN-D1795, Langley Research Center, Langley Station, Hampton, Virginia, March 1963.

19 Sommer, C.S. and Short, B.J., Free Flight Measurements of Turbulent-Boundary-Layer Skin Friction in the Presence of Severe Aerodynamic Heating at Mach Numbers from 2.8 to 7.0, NACA-TN-3391, Ames Aeronautical Laboratory, Moffett Field, California, March 1955. 